\title{
ÉDITORIAL
}

\section{Cancer du sein en 2010 : des avancées technologiques à une médecine encore plus personnalisée et humaine}

\author{
S. Delaloge \\ Département de médecine, institut Gustave-Roussy, 39-53, rue Camilles-Desmoulins, F-94805 Villejuif cedex, France \\ Correspondance : suzette.delaloge@igr.fr
}

Au tout début d'une nouvelle décennie très riche de promesses, ce dossier spécial d'oncologie met en lumière les avancées majeures des dernières, mais aussi des prochaines années en matière de prise en charge thérapeutique des femmes atteintes de cancer du sein.

Nous sommes à un tournant de l'oncologie pour lequel le cancer du sein est à l'avant-garde : les progrès récents, majeurs et rapides des technologies en matière de biologie, de génétique, d'imagerie, de radiophysique, de chirurgie permettent aujourd'hui de concrétiser nos espoirs et d'envisager une réelle prise en charge personnalisée sur les caractéristiques du cancer mais aussi sur les caractéristiques constitutionnelles de la personne.

Divers enjeux essentiels en thérapeutique sont largement évoqués ici : implémentation en routine des tests moléculaires pronostiques haut débit [4], développement des tests de seconde génération $[2,4,6]$, identification de nouvelles cibles thérapeutiques et développement des substances inhérentes [2], accès à une radiothérapie de pointe augmentant l'index thérapeutique [3], accès à une chirurgie personnalisée avec généralisation des pratiques d'oncoplastie [5], accès précoce à la recherche d'anomalies moléculaires constitutionnelles et choix thérapeutiques personnalisés qui en découlent [1].

Ces avancées technologiques complexes, fleurons de nos spécialités, ne doivent pas se dissocier d'une prise en charge encore plus humaine, plus à l'écoute, plus ouverte pour une pathologie si fréquente et touchant des femmes dans la force de l'âge. Les enjeux humains des avancées technologiques sont donc, en parallèle, discutés, en particulier dans l'après cancer [7] ou chez les femmes en situation de non-curabilité [6].

Nous vous souhaitons bonne lecture de ce passionnant et très documenté numéro d'Oncologie.

\section{Références}

Les articles référencés ci-après sont tous publiés dans ce dossier

1. Belkacémi Y, Tolédano A, Vieillot S, et al. (2010) Avancées en radiothérapie externe des cancers du sein. Oncologie 12(4): 269-73

2. Berrada N, André $F(2010)$ Traitements médicaux guidés par la biologie en pathologie mammaire. Oncologie 12(4): 274-77

3. Delaloge S, Rimareix F, Balleyguier C, et al. (2010) La génétique constitutionnelle peut-elle impacter la prise en charge d'une femme atteinte de cancer du sein aujourd'hui ? Oncologie 12(4): 248-54

4. Guiu S, Debled M (2010) Les grands challenges de la prise en charge en phase métastatique aujourd'hui. Oncologie 12(4): 278-84

5. Malka I, Salmon RJ, Couturaud B, et al. (2010) Technique chirurgicale : oncoplastie dans le traitement conservateur du cancer du sein. Oncologie 12(4): 255-62

6. Reyal F, Pierga JY, Salmon RJ, et al. (2010) Le point sur les signatures moléculaires dans le cancer du sein. Oncologie 12(4): 263-68

7. Zelek L, Bouillet T, Latino-Martel P, et al. (2010) Mode de vie et cancer du sein : quels conseils pour la prise en charge de l'après cancer? Oncologie 12(4): 289-97 